\title{
A CHARACTERIZATION OF SEMILINEAR SETS
}

\section{RANI SIROMONEY}

Introduction. The recent interest in the structure of programming languages has led to the study of their mathematical properties. Characterizations of bounded context-free languages (also called bounded ALGOL-like languages) [1] and bounded regular sets [3] have been given in terms of certain semilinear subsets of $N^{n}$. Semilinear sets have been extensively studied as subsets of lattice points in $n$-space which are finite unions of cosets of finitely generated subsemigroups of the set of all lattice points with nonnegative coordinates and which are also shown to be equivalent to the family of sets defined by modified Presburger formulas [2]. In this note we give a characterization and discuss decision procedures for semilinear sets of words (hereafter called semilinear sets) [4] which include bounded context-free languages and hence bounded regular sets.

1. Preliminaries. Let $\Sigma$ be a finite nonempty set and $\Sigma^{*}$ the free semigroup with identity $\epsilon$ generated by $\Sigma$. A subset $X$ of $\Sigma^{*}$ is said to be bounded if there exist words $w_{1}, \cdots, w_{k}$ in $\Sigma^{*}$ such that $X$ $\subseteq w_{1}^{*} \cdots w_{\mathbf{k}}^{*}$. For each $k$-tuple of words $w=\left\langle w_{1}, \cdots, w_{k}\right\rangle$ let $f_{w}$ denote the function defined on $N^{k}$ by $f_{w}(p)=w_{1}^{p_{1}^{(1)}} \cdots w_{\mathbf{k}}^{p(\mathbf{k})}$ where $p=(p(1), \cdots, p(k))$ is in $N^{k}$. Then $M \subseteq w_{1}^{*} \cdots w_{k}^{*}$ is said to be semilinear in $w$ if $w=\left\langle w_{1}, \cdots, w_{k}\right\rangle$ and $f_{w}{ }^{-1}(M)$ is a semilinear subset of $N^{k}$. A set $M$ is called semilinear if it is semilinear in some $k$-tuple $\left\langle w_{1}, \cdots, w_{k}\right\rangle[4]$.

An equal matrix grammar (abbreviated EMG) of order $k$ [5] is a 4-tuple $G=(V, \Sigma, P, S)$ where (i) $V$ consists of the alphabet $\Sigma$, the initial symbol $S$, and the rest of the nonterminals $V_{N}$ in the form of ordered $k$-tuples $\left\langle A_{1}, \cdots, A_{k}\right\rangle$ where the $k$-tuples are distinct, $k$ being finite. In other words if $\left\langle A_{1}, \cdots, A_{k}\right\rangle$ and $\left\langle B_{1}, \cdots, B_{k}\right\rangle$ are any two $k$-tuples, $A_{1}, \cdots, A_{k}, B_{1}, \cdots, B_{k}$ are distinct. (ii) $P$ consists of the following types of matrix rules:

(a) A set of initial matrix rules (abbreviated initial rules) of the form $\left[S \rightarrow f_{1} A_{1} \cdots f_{k} A_{k}\right]$ where $f_{1}, \cdots, f_{k}$ are in $\Sigma^{*}, S$ the initial symbol and $\left\langle A_{1}, \cdots, A_{k}\right\rangle$ in $V_{N}$. (Note that $S \rightarrow f_{1} A_{1} \cdots f_{k} A_{k}$ is a context-free rule.)

(b) A set of nonterminal equal matrix rules (abbreviated nonterminal rules) of the form

Received by the editors August 12, 1968. 


$$
\left[\begin{array}{c}
A_{1} \rightarrow f_{1} A_{1} \\
\cdot \cdot \cdot \cdot \\
A_{k} \rightarrow f_{k} A_{k}
\end{array}\right] \text { or }\left[\begin{array}{c}
A_{1} \rightarrow f_{1} B_{1} \\
\cdot \cdot \cdot \cdot \\
A_{k} \rightarrow f_{k} B_{k}
\end{array}\right]
$$

where $f_{1}, \cdots, f_{k}$ are in $\Sigma^{*}$ and $\left\langle A_{1}, \cdots, A_{k}\right\rangle,\left\langle B_{1}, \cdots, B_{k}\right\rangle$ in $V_{N}$.

(c) A set of terminal equal matrix rules (abbreviated terminal rules) of the form

$$
\left[\begin{array}{c}
A_{1} \rightarrow f_{1} \\
\cdot \cdot \cdot \\
A_{k} \rightarrow f_{k}
\end{array}\right]
$$

where $f_{1}, \cdots, f_{k}$ are in $\Sigma^{*},\left\langle A_{1}, \cdots, A_{k}\right\rangle$ in $V_{N}$. An equal matrix grammar is an EMG of any finite order.

Notation. Let $G=(V, \Sigma, P, S)$ be an EMG. We write $S \Rightarrow f_{1} A_{1}$ $\cdots f_{k} A_{k}$ if $\left[S \rightarrow f_{1} A_{1} \cdots f_{k} A_{k}\right]$ is an initial rule in $P$, and $w_{1} \Rightarrow w_{2}$ if $w_{1}=x_{1} A_{1} \cdots x_{k} A_{k}, w_{2}=x_{1} v_{1} \cdots x_{k} v_{k}, x_{i}$ in $\Sigma^{*},\left\langle A_{1}, \cdots, A_{k}\right\rangle$ in $V_{N}$ and

$$
\left[\begin{array}{c}
A_{1} \rightarrow v_{1} \\
\cdot \cdot \cdot \\
A_{k} \rightarrow v_{k}
\end{array}\right]
$$

is in $P$. We write $w \stackrel{*}{\Rightarrow} y$ if either $w=y$ or there exist $w_{0}=w, w_{1}, \cdots$, $w_{n}=y$ such that $w_{i} \Rightarrow w_{i+1}$ for each $i$. A sequence of words $w_{0}, \cdots, w_{n}$ such that $w_{i} \Rightarrow w_{i+1}$ for each $i$, is called a derivation or generation of $w_{n}$ (from $w_{0}$ ) and is denoted by $w_{0} \Rightarrow \cdots \Rightarrow w_{n} . L \subseteq \Sigma^{*}$ is an equal matrix language (abbreviated EML) if there is an EMG $G=(V, \Sigma, P, S)$ such that $L=L(G)$ where $L(G)=\left\{w\right.$ in $\left.\Sigma^{*} / S^{*} \Rightarrow w\right\} . L(G)$ is said to be the language generated by $G$.

2. Characterization. We now present a characterization of semilinear sets, which is related to Theorem 2.1 of [1] and Theorem 1.3 of $[3]$.

ThEOREM 2.1. $X \subseteq \Sigma^{*}$ is semilinear if and only if $X$ is a bounded EML.

Proof. Let $X$ be semilinear. Then there is a $w=\left\langle w_{1}, \cdots, w_{k}\right\rangle$ such that $X$ is semilinear in $w$, i.e. $L=\left\{(i(1), \cdots, i(k)) / w_{1}^{i(1)} \cdots w_{k}^{i(k)}\right.$ in $X\}$ is a semilinear subset of $N^{k}$. Let $a_{1}, \cdots, a_{k}$ be $k$ distinct symbols not in $\Sigma$ and $h$ the homomorphism which maps each $a_{i}$ into $w_{i}$. Then by Theorem 2.2 of [5], $Y=\left\{a_{1}^{i(1)} \cdots a_{k}^{i(k)} / w_{1}^{i(1)} \cdots w_{k}^{i(k)}\right.$ in $\left.X\right\}$ is an EML. By the corollary to Theorem 3.2 of [6] homomorphism preserves EML. Hence $X$ is a bounded EML. 
Now suppose $X$ be a bounded EML. $Y=h^{-1}(X) \cap a_{1}^{*} \cdots a_{k}^{*}$. By the corollary to Theorem 3.5 of [6] inverse homomorphism preserves EML and by Theorem 3.1 of [6] the intersection of an EML and a regular set is an EML. Hence $Y$ is an EML since $a_{1}^{*} \cdots a_{k}^{*}$ is regular. Again by Theorem 2.1 of [5], $L$ is a semilinear subset of $N^{k}$. Thus $X$ is semilinear.

Therefore the class of bounded EML is equivalent to the class of semilinear sets and includes the bounded context-free languages and hence the bounded regular sets.

Notation. Let $Z$ be a bounded set $\subseteq x_{1}^{*} \cdots x_{k}^{*}$, i.e. every $z$ in $Z$ is of the form $x_{1}^{i(1)} \cdots x_{k}^{i(k)}, x_{1}, \cdots, x_{k}$ being words in $\Sigma^{*}$. Then we write $Z\left\langle y_{1}, \cdots, y_{k}\right\rangle^{*}=\bigcup_{i \geq 0} z_{1} y_{1}^{i} z_{2} y_{2}^{i} \cdots z_{k} y_{k}^{i}$ where $y_{1}, \cdots, y_{k}$ are words in $x_{1}^{*}, \cdots, x_{k}^{*}$ respectively, and $z_{1}=x_{1}^{i(1)}, z_{2}=x_{2}^{i(2)}, \cdots, z_{k}=x_{k}^{i(k)}$ where $z=z_{1} \cdots z_{k}=x_{1}^{i(1)} \cdots x_{k}^{i(k)}$ is in $Z$. Inductively we write $Z\left\langle y_{11}, \cdots, \quad y_{k 1}\right\rangle \cdots\left\langle y_{1 n}, \cdots, \quad y_{k n}\right\rangle^{*}=Z\left\langle y_{11}, \cdots, \quad y_{k 1}\right\rangle \cdots$ $\left\langle y_{1 n-1}, \cdots, y_{k n-1}\right\rangle^{*}\left\langle y_{1 n}, \cdots, y_{k n}\right\rangle^{*}$ where $y_{11}, \cdots, y_{1 n}$ are words in $x_{1}^{*}, \cdots, y_{k 1}, \cdots, y_{k n}$ are words in $x_{k}^{*}$.

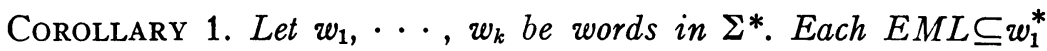
... $w_{\mathbf{k}}^{*}$ is the finite union of sets of the form

$$
x\left\langle y_{11}, \cdots, y_{k 1}\right\rangle \cdots\left\langle y_{1 n}, \cdots, y_{k n}\right\rangle^{*}
$$

where each $y_{r m}$ is in $w_{r}^{*}, r=1, \cdots, k ; m=1, \cdots, n$ and $x=x_{1} \cdots x_{k}$ where $x_{r}$ is in $w_{r}^{*}$; and conversely each finite union of sets of the above form is an $E M L \subseteq w_{1}^{*} \cdots w_{k}^{*}$.

COROLLARY 2. The family of bounded EML is the smallest family of sets containing all finite sets and closed with respect to the following operations:

(a) finite union,

(b) finite product,

(c) $Z\left\langle x_{1}, \cdots, x_{k}\right\rangle^{*}$ where $x_{1}, \cdots, x_{k}$ are words.

This is related to Theorem 3.1 of [1]. In view of Theorem 3.2 of [4] we obtain the following

Corollary 3. $S(L)$ is a bounded EML for each bounded EML $L$ and each gsm $S$.

3. Decidability. In this section, we consider the problem of determining of an arbitrary EML whether or not it is semilinear. We shall show that there is a decision procedure. Also another simple characterization of semilinear sets is given.

Notation. For each EMG $G$ of order $k$ and for each $k$-tuple of nonterminals $\left\langle A_{1}, \cdots, A_{k}\right\rangle$ let 


$$
\begin{aligned}
& X_{A_{1}}(G)=\left\{u_{1} / u_{1} \text { in } \Sigma^{*}, A_{1} \cdots A_{k} \stackrel{*}{\Rightarrow} u_{1} A_{1} \cdots u_{k} A_{k}\right. \\
& \text { for some } \left.u_{2}, \cdots, u_{k} \text { in } \Sigma^{*}\right\} \text {, } \\
& X_{A_{2}}(G)=\left\{u_{2} / u_{2} \text { in } \Sigma^{*}, A_{1} \cdots A_{k} \stackrel{*}{\Rightarrow} u_{1} A_{1} \cdots u_{k} A_{k}\right. \\
& \text { for some } \left.u_{1}, u_{3}, \cdots, u_{k} \text { in } \Sigma^{*}\right\} \text {, } \\
& X_{A_{k}}(G)=\left\{u_{k} / u_{k} \text { in } \Sigma^{*}, A_{1} \cdots A_{k} \stackrel{*}{\Rightarrow} u_{1} A_{1} \cdots u_{k} A_{k}\right.
\end{aligned}
$$$$
\text { for some } \left.u_{1}, \cdots, u_{k-1} \text { in } \Sigma^{*}\right\} \text {. }
$$

The results that follow are obtained by suitably modifying the methods of Ginsburg and Spanier [1].

LEMma 3.1. If $L(G)$ is nonempty and bounded where $G$ is of order $k$, then $X_{A_{1}}, \cdots, X_{A_{k}}$ are all commutative for each $k$-tuple $\left\langle A_{1}, \cdots, A_{k}\right\rangle$.

Proof. Let $G=(V, \Sigma, P, S)$ be the EMG generating $L$. Assume that $S$ depends on each $k$-tuple of nonterminals in $G$, and that $W_{A}$ $=\left\{w_{1} \cdots w_{k} / A_{1} \cdots A_{k} \stackrel{*}{\Rightarrow} w_{1} \cdots w_{k}, w_{r}\right.$ in $\left.\Sigma^{*}\right\}$ is nonempty for each $k$-tuple $\left\langle A_{1}, \cdots, A_{k}\right\rangle=A$ in $G$. Since $S$ depends on $A$, there exist $u_{1}, \cdots, u_{k}$ in $\Sigma^{*}$ such that $\left\{u_{1} w_{1} \cdots u_{k} w_{k} / w_{1} \cdots w_{k}\right.$ in $\left.W_{A}\right\}$ $\subseteq L(G)$. Thus $W_{A}$ is nonempty and bounded. Let $x_{1} \cdots x_{k}$ be a specific word in $W_{A}$.

Consider the set $X_{A_{1}}(G)$. Suppose there are words $u_{1}$ and $v_{1}$ in $X_{A_{1}}$ so that $u_{1} v_{1} \neq v_{1} u_{1}$. It is easily seen that for each $w_{1}$ in $\left\{u_{1}, v_{1}\right\}^{*}-\epsilon$ there are words $w_{2}, \cdots, w_{k}$ in $\Sigma^{*}$ so that $A_{1} \cdots A_{k} \stackrel{*}{\Rightarrow} w_{1} A_{1} \cdots w_{k} A_{k}$ $\stackrel{*}{\Rightarrow} w_{1} x_{1} \cdots w_{k} x_{k}$. Hence $\left(u_{1}, u_{2}\right)^{*}-\epsilon \subseteq X_{A_{1}}$ and $w_{1} x_{1} \cdots w_{k} x_{k}$ is in $W_{A}, \epsilon$ is also in $X_{A_{1}}$. Thus each word $w_{1} \cup_{\epsilon}$ in $\left\{u_{1}, u_{2}\right\} *$ is a subword of some word $w_{1} x_{1} \cdots w_{k} x_{k}$ in $W_{A}$. By Lemma 5.3 of [1], $W_{A}$ is not bounded. This is a contradiction. Therefore $u_{1} u_{2}=u_{2} u_{1}$ for every two words $u_{1}, u_{2}$ in $X_{A_{1}}(G)$ i.e. $X_{A_{1}}(G)$ is a commutative set.

A similar argument shows that $X_{A_{2}}(G), \cdots, X_{A_{k}}(G)$ are all commutative sets.

Lemma 3.2. If $X_{A_{1}}(G), \cdots, X_{A_{k}}(G)$ are all commutative sets for each $k$-tuple $\left\langle A_{1}, \cdots, A_{k}\right\rangle$ of $G$ of order $k$, then $L(G)$ is bounded.

Proof. The proof is by induction on the number of $k$-tuples of nonterminals. Suppose $\left\langle A_{1}, \cdots, A_{k}\right\rangle$ is the only nonterminal in $G$, apart from $S$. By Lemma 5.2 of $[1], X_{A_{1}} \subset u_{1}^{*}, \cdots, X_{A_{k}}{ }^{*} \subseteq u_{k}^{*}$ for some words $u_{1}, \cdots, u_{k}$ in $\Sigma^{*}$. Let all the initial and terminal rules of $G$ be $\left[S \rightarrow f_{1 j} A_{1} \cdots f_{k j} A_{k}\right], j=1, \cdots, m$; 


$$
\left[\begin{array}{c}
A_{1} \rightarrow w_{1 i} \\
\cdot \cdot \cdot \\
A_{k} \rightarrow w_{k i}
\end{array}\right] \quad i=1, \cdots, n .
$$

If $y$ be any word in $L(G)$, there is some $S$-derivation of $y$ as $S \Rightarrow f_{1 j} A_{1}$ $\cdots f_{k j} A_{k} \stackrel{*}{\Rightarrow} f_{1 j} v_{1} A_{1} \cdots f_{k j} v_{k} A_{k} \Rightarrow f_{1 j} v_{1} w_{1 i} \cdots f_{k j} v_{k} w_{k i}, \quad 1 \leqq j \leqq m, \quad 1 \leqq i$ $\leqq n, v_{1}, \cdots, v_{k}$ in $X_{A_{1}}, \cdots, X_{A_{k}}$ which are subsets of $u_{1}^{*}, \cdots, u_{k}^{*}$. Thus

$$
L(G) \subseteq \bigcup_{j=1}^{m}\left[\bigcup_{i=1}^{n} f_{1 j} u_{1}^{*} w_{1 i} \cdots f_{k j} u_{k}^{*} w_{k i}\right]
$$

Therefore $L(G)$ is bounded.

Suppose that $G$ has $p k$-tuples of variables $\left\langle A_{1 i}, \cdots, A_{k i}\right\rangle, i$ $=1, \cdots, p$, where $p>1$ and that the lemma is true for all grammars with fewer than $p$ variables. Let $G_{j}$ be the grammar obtained from $G$ by deleting all the production rules involving $\left\langle A_{1 j}, \cdots, A_{k j}\right\rangle$. Let $Y_{A_{1 i}}\left(G_{j}\right), \cdots, Y_{A_{k i}}\left(G_{j}\right)$ be the set of words $y_{1 i}, \cdots, y_{k i}$ such that $A_{1 i} \cdots A_{k i} \stackrel{*}{\Rightarrow} G_{j} y_{1 i} \cdots y_{k i}$ in $\Sigma^{*}$. $X_{A_{1 i}}\left(G_{j}\right), \cdots, X_{A_{k i}}\left(G_{j}\right)$ being subsets of $X_{A_{1 i}}(G), \cdots, X_{A_{k i}}(G)$ are all bounded. By the induction hypothesis $L\left(G_{j}\right)$ is bounded. $Y_{A_{1 i}}\left(G_{j}\right), \cdots, Y_{A_{k i}}\left(G_{j}\right)$ consisting of subwords of words in $L\left(G_{j}\right)$ are bounded. Let there be $q$ initial rules $\left[S \rightarrow f_{1 j} A_{1 j} \cdots f_{k j} A_{k j}\right], j=1, \cdots, q$. For each such $j$, consider

$$
f_{1 j} X_{A_{1 j}}(G) g_{1 i} Y_{A_{1 i}}\left(G_{j}\right) \cdots f_{k j} X_{A_{k j}}(G) g_{k i} Y_{A_{k i}}\left(G_{j}\right),
$$

$i$ in $\{1, \cdots, p\}-\{j\}$ where

$$
\left[\begin{array}{c}
A_{1 j} \rightarrow g_{1 i} A_{1 i} \\
\cdot \cdot \cdot \cdot \\
A_{k j} \rightarrow g_{k i} A_{k i}
\end{array}\right]
$$

are all the rules of $G$ with $\left\langle A_{1 j}, \cdots, A_{k j}\right\rangle$ occurring on the left side. (When the above rule is terminal, the $Y$ 's are empty.) Since there are only a finite number of such rules the sets $\left({ }^{* *}\right)$ are bounded. The proof is completed by noting that

$$
L(G) \subseteq \bigcup_{j=1}^{q} f_{1 j} X_{A_{1 j}} g_{1 i} Y_{A_{1 i}} \cdots f_{k j} X_{A_{k j}} g_{k i} Y_{A_{k i}}
$$

Combining Lemmas 2.1 and 2.2 we get

TheOREM 3.1. A necessary and sufficient condition that an EML 
$L(G) \neq \varnothing$ be semilinear is that $X_{A_{1}}(G), \cdots, X_{A_{k}}(G)$ be all commutative for each variable $\left\langle A_{1}, \cdots, A_{k}\right\rangle$ in $G$ of order $k$.

Lemma 3.3. For each variable $\left\langle A_{1}, \cdots, A_{k}\right\rangle$ in $G$ of order $k, X_{A_{1}}(G)$, $\cdots, X_{A_{k}}(G)$ are regular sets and effectively determined.

The proof is obvious from the definition of an EMG that all rules except the initial rules consist of $k$ left-linear rules.

Now from Lemma 2.3, and Lemmas 5.7 and 5.8 of [1] and the proof of Lemma 2.2, the following decision theorem is immediate.

THEOREM 3.2. (a) It is decidable whether or not a given $E M L L(G)$ is bounded.

(b) If $L(G)$ is bounded then words $w_{1}, \cdots, w_{t}$ in $\Sigma^{*}$ can be effectively found so that $L(G) \subseteq w_{1}^{*} \cdots w_{1}^{*}$.

THEOREM 3.3. If $L_{1}, L_{2}$ are EML and one of them is semilinear, then it is solvable whether (a) $L_{1} \subseteq L_{2}$ and whether (b) $L_{2} \subseteq L_{1}$.

Proof is immediate from the proof of Theorem 6.3 of [1] using the corresponding results for EML obtained in Theorems 2.2 and 1.1.

COROLLARY. If $L_{1}, L_{2}$ are EML and one of them is semilinear then it is solvable whether $L_{1}=L_{2}$.

Several of the mathematical properties of semilinear sets proved in [4] can also be established by considering bounded EML.

\section{REFERENCES}

1. S. Ginsburg and E. H. Spanier, Bounded ALGOL-like languages, Trans. Amer. Math. Soc. 113 (1964), 333-368.

2. - Semigroups, Presburger formulas, and languages, Pacific J. Math. 16 (1966), 285-296.

3. - Bounded regular sets, Proc. Amer. Math. Soc. 17 (1966), 1043-1049.

4. S. Ginsburg and J. Ullian, Ambiguity in context free languages, J. Assoc. Comput. Mach. 13 (1966), 62-89.

5. R. Siromoney, On equal matrix languages, Information and Control, (to appear).

6. - k-turn one-way stack automata, (to appear).

Madras Christian College, India 